\title{
miRNA299 involvement in CYP11B2 expression in aldosterone-producing adenoma
}

\author{
Yujiro Nakano', Takanobu Yoshimoto', Ryo Watanabe², Masanori Murakami', Tatsuya Fukuda', \\ Kazutaka Saito ${ }^{3}$, Yasuhisa Fujiii ${ }^{3}$, Takumi Akashi ${ }^{4}$, Toshihiro Tanaka², Tetsuya Yamada', \\ Mitsuhide Naruse ${ }^{5}$ and Yoshihiro Ogawa ${ }^{6,7}$
}

1Department of Molecular Endocrinology and Metabolism, Graduate School of Medical and Dental Sciences, Tokyo Medical and Dental University, Tokyo, Japan, 2Department of Human Genetics and Disease Diversity, Tokyo Medical and Dental University, Tokyo, Japan, ${ }^{3}$ Department of Urology, Graduate School of Medical and Dental Sciences, Tokyo Medical and Dental University, Tokyo, Japan, ${ }^{4}$ Department of Pathology, Tokyo Medical and Dental University, Tokyo, Japan, ${ }^{5}$ Department of Endocrinology, Metabolism and Hypertension, National Hospital Organization, Kyoto Medical Center, Kyoto, Japan, ${ }^{6}$ Department of Molecular and Cellular Metabolism, Graduate School of Medical and Dental Sciences, Tokyo Medical and Dental University, Tokyo, Japan, and ${ }^{7}$ Department of Medicine and Bioregulatory Science, Graduate School of Medical Sciences, Kyushu University, Fukuoka, Japan

Correspondence should be addressed to T Yoshimoto Email

tyoshimoto.mem@tmd.ac.jp

\begin{abstract}
Objective: The pathophysiology of aldosterone-producing adenomas (APAs) has been intensively investigated using genetic and epigenetic approaches. However, the role of miRNAs in APA is not fully understood. The present study profiled miRNAs in APAs as an exploratory approach to elucidate their pathophysiological roles in APAs. Design: Tissues of APAs and other adrenocortical adenomas were obtained from patients who underwent adrenalectomy.

Methods: Candidate miRNAs differentially detected from samples were examined by whole miRNA sequencing. The expression of candidate miRNAs in APA tissues were further validated by real-time quantitative polymerase chain reaction (qPCR). Further, differential miRNA expression between APAs with and without KCNJ5 somatic mutations was examined. Prediction of miRNA target genes was performed by bioinformatics analysis. For specific miRNAs, correlation analysis between the levels of their target genes and CYP11B2 was analyzed in APA tissues.

Results: Our study determined differential expression of six miRNAs in APA or APA with KCNJ5 mutations. We further demonstrated that miR299 levels were negatively correlated with mRNA levels of CACNB2, which encodes the betasubunit of the L-type calcium channel. Additionally, we found significant correlations among miR299, CACNB2, and CYP11B2 levels in APA tissues.

Conclusions: Our study suggests the possible pathophysiological involvement of specific miRNAs in calcium signaling and aldosterone hypersecretion in APAs. Further studies, including in vitro analyses, are required to clarify these findings.
\end{abstract}

\section{Introduction}

Primary aldosteronism (PA) is an important cause of secondary hypertension $(1,2)$ and has been associated with severe cardiovascular and renal complications, as shown by comparison with age-, sex- and blood pressurematched patients with essential hypertension $(3,4)$. Among the PA subtypes, aldosterone-producing adenoma (c) 2019 European Society of Endocrinology Printed in Great Britain
(APA) often causes severe forms of resistant hypertension that are curable by unilateral adrenalectomy, making APAs therapeutically attractive $(1,5)$.

Recently, the pathogenesis of APA has been clarified at the molecular level using high-throughput technologies, including next-generation sequencing, transcriptomics 
and methylomics $(6,7,8)$. In particular, a series of exome sequencing studies have uncovered that somatic mutations in genes encoding ion channels or carriers (KCNJ5, ATP1A1, ATP2B3 and CACNA1D) $(9,10,11,12,13)$ cause activation of calcium (Ca) signaling. This activation leads to autonomous aldosterone hypersecretion from APA through increased expression of CYP11B2, a gene encoding a steroidogenic enzyme responsible for a ratelimiting step of aldosterone synthesis (14). Furthermore, methylome studies have revealed that hypomethylation of the proximal promoter region of CYP11B2 is involved in its increased expression in $\operatorname{APA}(15,16)$. Thus, the pathophysiology and pathogenesis of APA are currently being clarified in both genomic and epigenomic studies.

miRNA, another important epigenetic factor, is a small non-coding RNA molecule. It is widely accepted that miRNAs bind to their target mRNAs through their complementary sequences $(17,18)$ and negatively regulate the expression of their target gene through mRNA degradation and post-transcriptional modification (19). A series of studies have shown that miRNAs are crucial regulators of biological processes, including various pathological conditions, such as neoplasms $(20,21)$. However, to date, data on miRNA expression in APAs are limited.

In the present study, we profiled miRNAs that are differentially detected in tumor tissues from patients with APAs via whole miRNA sequencing, as an exploratory approach to elucidate the pathophysiological significance of miRNAs in APAs. The differential expression of miRNA between APAs with or without KCNJ5 somatic mutations was also examined.

\section{Subjects and methods}

\section{Subjects}

The study protocol was approved by the Ethics Committee for Human Research at Tokyo Medical and Dental University (Tokyo, Japan) (nos. 191 and G2016002). Written informed consent was obtained from all participants. Adrenal tissue was obtained from 62 patients with APA, 12 patients with adrenocortical adenoma (ACA) with subclinical hypercortisolism (SH) and four patients with non-functioning adrenal adenoma (NF) who underwent adrenalectomy at Tokyo Medical and Dental University Hospital as described previously (8). Among them, 12 APAs and 5 SHs were from cases that were used in previous studies (8).
The diagnosis of PA and SH was based on diagnostic criteria as described in the Supplementary materials (see section on supplementary data given at the end of this article). NF patients did not fulfill diagnosis criteria of either PA or SH. All adrenal tumors were pathologically diagnosed as benign adrenocortical adenoma.

\section{Detection of somatic mutations in KCNJ5, ATP1A1, ATP2B3, CACNA1D and CTNNB1 by PCR and direct sequencing}

APA-specific somatic mutations in KCNJ5, ATP1A1, ATP2B3, CACNA1D and CTNNB1 were analyzed by PCR and direct sequencing. The detailed methods have been described previously (22), and the primers used are described in Supplementary Table 1.

\section{Extraction of RNA}

Adrenocortical tumor samples were obtained at the time of surgery and stored at $-80^{\circ} \mathrm{C}$. Detailed protocols of RNA extraction are provided in the Supplementary materials.

\section{Whole miRNA sequencing and differential expression analysis}

A total of 14 adrenocortical tumors from patients with APA, 5 patients with SH and three patients with NF were investigated by whole miRNA sequencing as 'profiling samples'. Following RNA extraction, 500-1000ng of total RNA were used for whole miRNA sequencing and subsequent differential expression analysis. Detailed protocols are described in the Supplementary materials.

Hierarchical cluster analysis using whole miRNA sequence data showed that SHs clustered more closely to NFs than APAs (Supplementary Fig. 1). Due to the similarity of miRNA distributions of SHs and NFs, we set a combination of SHs and NFs as a control group for the subsequent analysis.

To search for miRNAs potentially involved in the pathogenesis of APAs, we defined the following profiling criteria to screen miRNAs by whole miRNA sequencing: Criterion (1) differentially detected miRNAs (adjusted $P$ value $<0.05$ ) in APA tissues compared to the control ( $\mathrm{SH}$ and NF tissues) and Criterion (2) differentially detected miRNAs (adjusted $P$ value $<0.05$ ) between APAs with and without KCNJ5 somatic mutations (Fig. 1). 


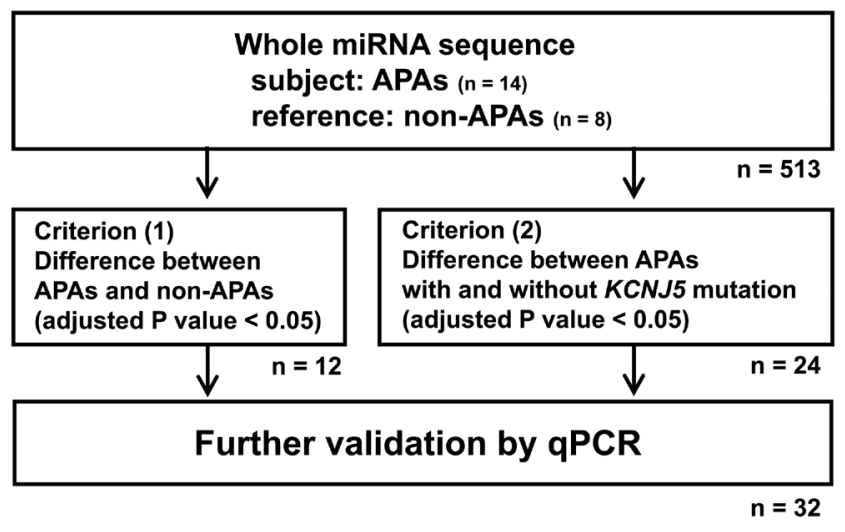

\section{Figure 1}

Strategy for miRNA profiling by whole miRNA sequencing. A total of 14 APAs (9 with KCNJ5 mutation and 5 without KCNJ5 mutation) and 8 non-APAs (5 SHs and 3 NFs) were examined as 'profiling samples'. Using whole miRNA sequencing, 513 miRNAs were detected; of which, 12 miRNAs met criterion 1 and 24 miRNAs met criterion 2. Four miRNAs met both criteria 1 and 2 . These 32 miRNAs are listed in Supplementary Table 5. APA, aldosteroneproducing adenoma; miRNA, microRNA; n, numbers of detected miRNAs; NF, non-functioning adrenal adenoma; qPCR, real-time quantitative polymerase chain reaction; $\mathrm{SH}$, adrenocortical adenoma with subclinical hypercortisolemia.

\section{Real-time qPCR analyses of miRNAs}

To validate results of the whole miRNA sequencing, reverse transcription and qPCR were performed as described in the Supplementary materials. For qPCR analysis, ten patients with APA, seven patients with SH and one patient with NF were added to the 'profiling samples' used for whole miRNA sequencing. Accordingly, 24 patients with APA, 12 patients with SH and four patients with NF were investigated as 'validation samples'.

\section{Prediction of miRNA target genes}

Prediction of miRNA target genes was performed by bioinformatics analysis using an online tool (http://www. mirdb.org/miRDB/). First, candidate target genes with a target score $>75$ were extracted from the miRDB database. We postulated that if the miRNA controlled expression of the target gene, the expression level of the miRNA and target gene would be inversely correlated. The candidate target genes were, therefore, narrowed down using the gene expression profiles of our previously published transcriptome database based on RNA sequencing (https://doi.org/10.1530/ERC-17-0117) (8). Selected genes consisted of 34 APAs (29 with and 5 without KCNJ5 mutations) and $5 \mathrm{SHs}$, as follows. For miR34a, which corresponds to Criterion 1, we selected genes showing greater than two-fold mRNA expression in APAs than $\mathrm{SH}$ in the RNA sequence database. For miR370, which corresponds to Criterion 1, we selected genes showing lesser than two-fold mRNA expression in APAs than SH in the RNA sequence database. For miR24, miR299, miR30e and miR93, which correspond to Criterion 2, we selected genes showing greater than two-fold mRNA expression in APAs with KCNJ5 mutations than APAs without KCNJ5 mutations in the RNA sequence database. The correlation of expression levels between these genes predicted by bioinformatics analysis and the corresponding miRNAs were further examined by qPCR as described below.

\section{Reverse transcription and qPCR analyses for gene expression}

For mRNA quantification in adrenocortical tumor specimens, reverse transcription and $\mathrm{qPCR}$ analysis were performed for the 29 candidate target genes by the method described previously (22). Notably, to examine the relation of $C A C N B 2$ gene expression with miRNA and CYP11B2, we analyzed 62 APA samples (43 APA with KCNJ5 mutations and 19 APA without KCNJ5 mutations) as 'confirmation samples'. The detailed protocols and primers used for the qPCR assay are described in the Supplementary materials.

\section{Statistical analysis}

Statistical analysis was carried out using SPSS (version 21.0; IBM Corp). Data are presented as the median and interquartile range (IQR). Due to skewed distribution, miRNA levels of miR370, miR24 and miR93 and mRNA levels of NEFM, GRIP1, FRMD6, HSPB7, CACNB2, CTNND2, CHST11, HSPA12A, TMEM154 and CYP11B2 were log-transformed for statistical analysis. In the whole miRNA sequence, adjusted $P$ values were calculated using the Benjamini and Hochberg false discovery rate correction as a reference. The hierarchical cluster analysis was performed by edgeR (v.3.18.1) (23). Mann-Whitney $U$ test was used to compare quantitative variables between two groups. Univariate correlations were evaluated as Pearson's correlation coefficient, since each parameter was normally distributed. Adjusted $P$-value $<0.05$ in the whole miRNA sequence and $P$ values $<0.05$ in the qPCR were considered to be statistically significant. 


\section{Results}

\section{Profiling of tissue miRNAs in patients with PA}

Clinical characteristics of the APA, SH and NF patients were divided into profiling samples, validation samples, and confirmation samples, as described in Table 1. Profiling revealed KCNJ5 somatic mutations in $70 \%$ of APA specimens. The somatic mutational state of APAs in the present study is described in Supplementary Table 4.

Our strategy for miRNA profiling by whole miRNA sequencing is shown in Fig. 1. In analysis of adrenocortical adenomas, 513 miRNAs were detected. Of which, 12 miRNAs met Criterion 1, and 24 miRNAs met Criterion 2. Four miRNAs (miR1307, miR370, miR532 and miR675) met both Criteria 1 and 2. These 32 miRNAs are listed in Supplementary Table 5.

\section{Validation of miRNAs profiling by qPCR in adrenocortical adenoma samples}

To validate the results of whole miRNA sequencing, we analyzed the levels of these 32 miRNAs in adrenocortical adenoma samples by qPCR (Supplementary Table 5 ). The levels of six miRNAs (miR24, miR299, miR30e, miR34a, miR370 and miR93) showed significant differential expression, which corresponded with the whole miRNA sequencing data (Fig. 2 and Supplementary Table 6). The levels of miR34a and miR370 were significantly lower and higher, respectively, in APA than in the reference control (NFs and SHs), which aligned with the 12 miRNAs of Criterion 1. Further, the levels of miR24, miR299, miR30e and miR93 were significantly lower in APAs with KCNJ5 mutations than in those without KCNJ5 mutations, which aligned with the 24 miRNAs of Criterion 2 (Fig. 2).

Table 1 Clinical characteristics of ACA patients. All data are presented as the median and interquartile range. 'Profiling samples', 'validation samples' and 'confirmation samples' indicate samples used for whole miRNA sequencing, qPCR for validation of miRNA expression and validation of the correlation between miRNAs and target genes, respectively. Validation samples contain the all of 'Profiling samples' and confirmation samples contain all APAs of 'Validation samples'. Hypokalemia was defined by serum potassium $<3.5 \mathrm{mEq} / \mathrm{L}$ or by treatment with any oral potassium supplement.

Profiling samples
Number
Age
Female (\%)
Duration of HT (Y)
Hypokalemia (\%)
PRA (ng/dL/h)
PAC (pg/mL)
Validation samples
Number
Age
Female (\%)
Duration of HT (Y)
Hypokalemia (\%)
PRA (ng/dL/h)
PAC (pg/mL)
Confirmation samples
Number
Age
Female (\%)
Duration of HT (Y)
Hypokalemia (\%)
PRA (ng/dL/h)
PAC (pg/mL)

\begin{tabular}{|c|c|}
\hline \multicolumn{2}{|c|}{ Non-APA } \\
\hline $\mathrm{NF}$ & $\mathrm{SH}$ \\
\hline 3 & 5 \\
\hline $63(60-66)$ & $52(46-54)$ \\
\hline 33 & 80 \\
\hline $0(0-4)$ & $0(0-13)$ \\
\hline 0 & 0 \\
\hline $0.6(0.5-2.1)$ & $0.2(0.2-0.3)$ \\
\hline $77.0(59.5-79.5)$ & $76.0(71.0-84.0)$ \\
\hline 4 & 12 \\
\hline $60(57-64)$ & $50(45-54)$ \\
\hline 25 & 92 \\
\hline $0(0-2)$ & $1(0-5)$ \\
\hline 0 & 25 \\
\hline $0.5(0.3-1.3)$ & $0.3(0.2-1.0)$ \\
\hline 79.5 (68.2-89.7) & $77.0(68.7-91.0)$ \\
\hline - & - \\
\hline - & - \\
\hline - & - \\
\hline - & - \\
\hline- & - \\
\hline - & - \\
\hline - & - \\
\hline
\end{tabular}

\begin{tabular}{|c|c|}
\hline \multicolumn{2}{|c|}{ APA } \\
\hline Non-KCNJ5 & KCNJ5 \\
\hline 5 & 9 \\
\hline $62(57-62)$ & $54(54-60)$ \\
\hline 20 & 77 \\
\hline $16(7-17)$ & $7(2-12)$ \\
\hline 100 & 88 \\
\hline $0.2(0.2-0.7)$ & $0.1(0.1-0.3)$ \\
\hline 283 (258-395) & $317(285-457)$ \\
\hline 11 & 13 \\
\hline $57(49-62)$ & $54(50-57)$ \\
\hline 37 & 69 \\
\hline 15 (7-19) & $9(3-12)$ \\
\hline 100 & 84 \\
\hline $0.2(0.1-0.5)$ & $0.2(0.1-0.3)$ \\
\hline 241 (198-367) & $326(289-413)$ \\
\hline 19 & 43 \\
\hline $57(49-62)$ & $51(42-57)$ \\
\hline 27 & 71 \\
\hline $9(6-16)$ & $9(4-15)$ \\
\hline 83 & 94 \\
\hline $0.2(0.2-0.6)$ & $0.2(0.1-0.3)$ \\
\hline $270(198-416)$ & $403(273-491)$ \\
\hline
\end{tabular}

ACA, adrenocortical adenoma; APA, aldosterone-producing adenoma; $\mathrm{HT}$, hypertension; $\mathrm{K}$, serum potassium; NF, non-functioning adrenal adenoma; Non-KCNJ5, APA without KCNJ5 somatic mutations; PAC, plasma aldosterone concentration; PRA, plasma renin activity; SH, ACA with subclinical hypercortisolemia. 
*: $P<0.05$
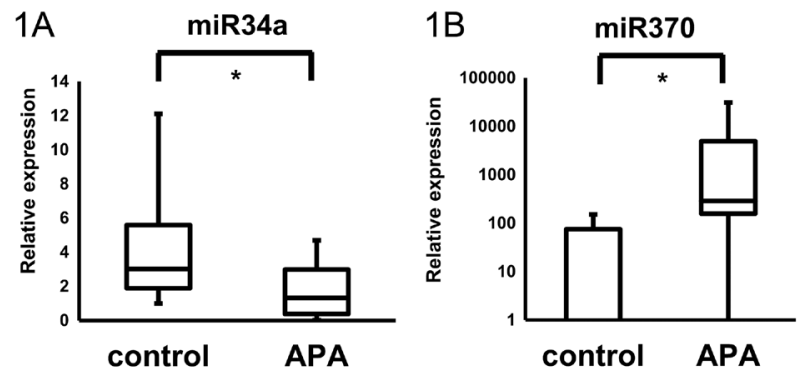

$2 \mathrm{~A}$
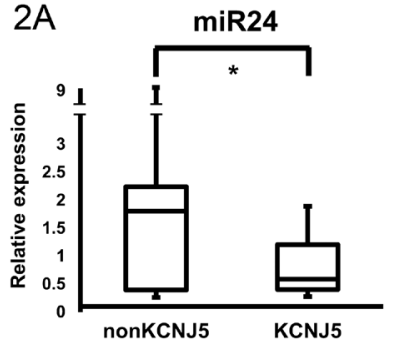

2B

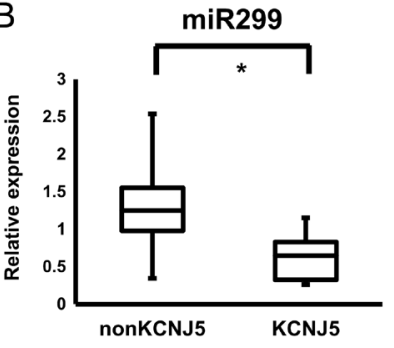

$2 \mathrm{C}$
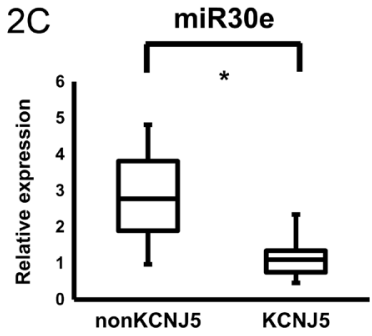

2D

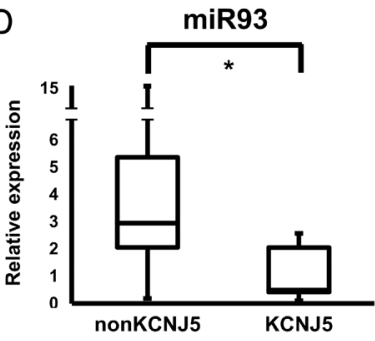

\section{Figure 2}

Differential expression of miRNAs in APA or APA with KCNJ5 somatic mutations validated by qPCR. Data were analyzed by qPCR. Relative miRNA levels were calculated and are presented as box plots. Controls consisted of 12 adrenocortical adenomas with subclinical hypercortisolemia and four non-functioning adrenal adenomas. APA consisted of 24 APA specimens regardless of KCNJ5 somatic mutation status. Non-KCNJ5 consisted of 11 APAs without KCNJ5 somatic mutations. KCNJ5 consisted of 13 APAs with $K C N J 5$ somatic mutations. $P$ values were calculated using the Mann-Whitney $U$ test. * indicates $P<0.05$ between two groups. APA, aldosterone-producing adenoma; KCNJ5, APA with KCNJ5 somatic mutations; miRNA, microRNA; non-KCNJ5, APA without KCNJ5 somatic mutations; qPCR, real-time quantitative polymerase chain reaction.

The expression of miRNAs profiled by Criterion 1 in the whole miRNA sequence was investigated by qPCR to validate use of the SH and NF combination as a reference control to APA. The qPCR analysis revealed that the distribution of all miRNAs, except miR34, was not significantly different between SHs and NFs (Supplementary Fig. 2).

\section{Nine target genes negatively correlated with levels of the corresponding miRNAs}

Integration of bioinformatics analysis using the online tool and our transcriptome database (8) predicted 29 candidate genes that are possibly regulated by the six miRNAs (Supplementary Table 7). We examined mRNA levels of these 29 genes by qPCR using the validation samples and then analyzed the correlation between expression levels of the miRNAs and their target genes (Table 2). The mRNA levels of CACNB2, which encodes L-type Ca channel and could potentially affect cellular Ca signaling, showed a significantly negative correlation to miR299 (Table 2). The mRNA levels of CTNND2, ANK2, RIMS3, MSTN, LRRK1, MAP3K8, GRIP1 and PPP4R4 also showed significant correlation to the corresponding miRNA levels (Table 2). The mRNA levels of the other genes showed no significant correlation to the corresponding miRNA levels (Table 2).

\section{Expression levels of CACNB2 positively correlated with CYP11B2}

CACNB2 encodes the $\beta 2$ subunit of the L-type Ca channel, which interacts with the $\alpha 1$ subunit to modulate function of the Ca channel $(24,25)$. Dysregulation of Ca signaling in adrenocortical cells is considered as one of the crucial pathogenic factors leading to CYP11B2 upregulation and subsequent autonomous aldosterone hypersecretion in PA. We therefore further examined the relationship of CACNB2 mRNA levels with those of CYP11B2 using the confirmation samples. The mRNA levels of CACNB2 in the confirmation samples showed significant negative correlations to miR299 levels (Fig. 3). Moreover, CACNB2 mRNA levels showed a significantly positive correlation with CYP11B2 (Fig. 3).

We also compared the miR299 levels between the entire APA and the control as a secondary sub-analysis using validation samples, although this comparison did not show significant difference in the miRNA profiling conducted using whole miRNA sequencing using the profiling samples. We found that the levels of miR299 were also significantly lower in the entire APA than in the controls using the validation samples (Supplementary Fig. 3). The mRNA levels of CACNB2 showed significant negative and positive correlations to miR299 levels and CYP11B2 mRNA levels, respectively, in the entire ACA that consist of the confirmation samples and controls (Supplementary Fig. 3). 
Table 2 Correlations between miRNA and gene expression levels of candidate genes profiled by bioinformatics analysis. Pearson's correlation coefficient was evaluated in each combination of miRNAs and candidate target genes, which were shown by bioinformatics analysis. Only the significant genes are described by their gene description.

\begin{tabular}{|c|c|}
\hline miRNA & Gene symbol \\
\hline \multicolumn{2}{|c|}{ APA compared with other ACA } \\
\hline \multirow[t]{7}{*}{ hsa-miR-34a-5p } & BMP3 \\
\hline & CTNND2 \\
\hline & TPD52 \\
\hline & $R E L N$ \\
\hline & ANK2 \\
\hline & RIMS3 \\
\hline & MSTN \\
\hline \multirow[t]{7}{*}{ hsa-miR-370-3p } & LRRK1 \\
\hline & CHST15 \\
\hline & CHST11 \\
\hline & HSPA12A \\
\hline & PMAIP1 \\
\hline & TMEM154 \\
\hline & МАРЗК \\
\hline \multicolumn{2}{|c|}{ APA with $K C N J 5$ mutations compared with those } \\
\hline \multirow[t]{3}{*}{ hsa-miR-24-3p } & NEFM \\
\hline & PTPRD \\
\hline & GRIP1 \\
\hline \multirow[t]{4}{*}{ hsa-miR-299-3p } & FRMD6 \\
\hline & $P A I P 2 B$ \\
\hline & HSPB7 \\
\hline & CACNB2 \\
\hline \multirow[t]{5}{*}{ hsa-miR-30e-5p } & PPP4R4 \\
\hline & GRM3 \\
\hline & CACNB2 \\
\hline & CTNND2 \\
\hline & ELOVL7 \\
\hline \multirow[t]{3}{*}{ hsa-miR-93-3p } & CACNB2 \\
\hline & ATP9A \\
\hline & $D L G A P 1$ \\
\hline
\end{tabular}

\begin{tabular}{c}
\hline $\boldsymbol{r}$ \\
\hline-0.350 \\
-0.442 \\
-0.343 \\
-0.174 \\
-0.629 \\
-0.467 \\
-0.456 \\
-0.589 \\
-0.387 \\
-0.338 \\
-0.372 \\
-0.267 \\
-0.332 \\
-0.417
\end{tabular}

P value

Gene description

0.054

$\mathbf{0 . 0 1 3}$

0.059

0.437

$<0.001$

0.008

0.011

0.010

0.102

0.512

0.117

0.269

0.227

$\mathbf{0 . 0 3 8}$ MAP kinase kinase kinase $8(41,42)$

thout mutations

$P$ values $<0.05$ are indicated in bold.

ACA, adrenocortical adenoma; APA, aldosterone-producing adenoma; $r$, correlation coefficient.

\section{mRNA levels of CYP11B2 correlated with miR299}

We examined the correlation of CYP11B2 mRNA levels with levels of miR299 using the confirmation samples. CYP11B2 mRNA levels showed significant negative correlations with miR299 levels (Fig. 4). CYP11B2 mRNA levels in the entire ACA that consist of the confirmation samples and controls also showed significant negative correlations with miR299 levels (Supplementary Fig. 3).

\section{Discussion}

We identified six miRNAs $(24,299,30 e, 34 a, 370$ and 93) by a combination of whole miRNA sequencing and qPCR. In addition, we revealed that miR299 levels in APA tissues were inversely correlated with CACNB2 expression levels. Furthermore, we revealed that $C Y P 11 B 2$ expression levels in APA tissues were positively and negatively correlated with CACNB2 and miR299 expression levels, respectively, suggesting pathophysiological relevance of the relationship among miR299, CACNB2, and CYP11B2.

The two analytical methods for miRNA, whole miRNA sequencing and qPCR, have several advantages and disadvantages. Whole miRNA sequencing is capable of comprehensively profiling miRNA expression by processing a large number of samples. However, qPCR can accurately quantify individual miRNAs, although it is difficult to profile a large number of miRNAs. Therefore, in the present study, we first screened samples using whole miRNA sequencing and then validated the obtained candidate miRNAs using qPCR. We screened 32 candidate miRNAs by whole miRNA sequencing and validated six 
A
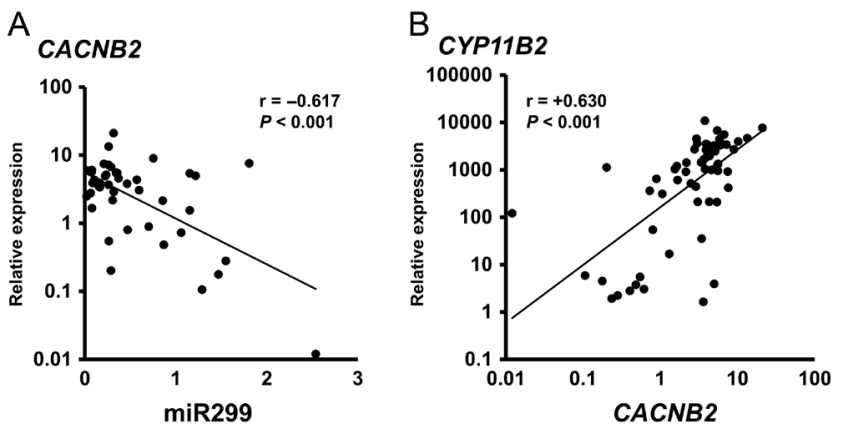

Figure 3

Correlations between CACNB2 mRNA levels and miR299 or CYP11B2 mRNA levels. Univariate correlations between CACNB2 mRNA levels and miR299 expression levels (A), and CYP11B2 mRNA levels (B) are shown. Pearson's correlation coefficient was used to analyze 62 APAs regardless of KCNJ5 somatic mutation status. APA, aldosterone-producing adenoma; $r$, correlation coefficient.

miRNAs by qPCR. Similar to the present study, Tiedt et al. comprehensively examined circulating miRNAs in patients suffering from acute ischemic stroke using whole miRNA sequencing and qPCR and reported that six miRNAs were validated by qPCR from 22 miRNAs profiled by whole

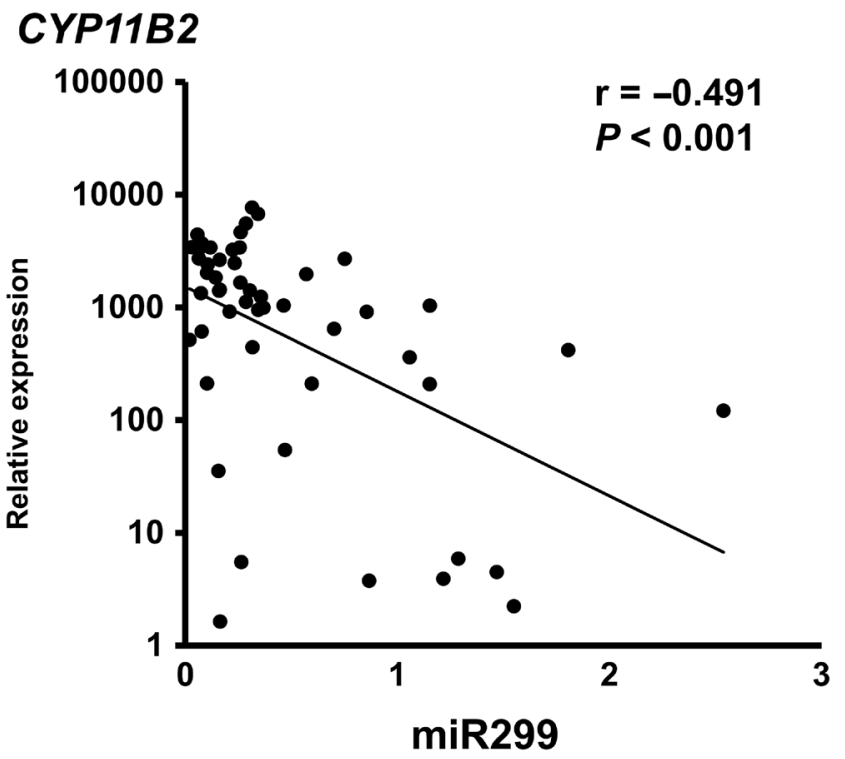

\section{Figure 4}

Correlations between miR299 levels and CYP11B2 mRNA levels. Univariate correlations between CYP11B2 mRNA levels and miR299 expression levels are shown. Pearson's correlation coefficient was used to analyze 62 APAs regardless of KCNJ5 somatic mutation status. APA, aldosterone-producing adenoma; $r$, correlation coefficient.
miRNA sequencing (26). Therefore, consistency between results of whole miRNA sequencing and qPCR does not appear to be high. In a different viewpoint, although our whole miRNA sequence did not show the significant difference in miR299 levels between APAs and controls, the qPCR showed a significant difference between them. It is, therefore, possible to speculate that the miRNA profiling method that we adopted overlooked some other miRNAs that are relevant in the pathophysiology of APA. Further studies are required to develop a more efficient screening method to examine miRNAs relevant to disease models.

Of the six miRNAs validated by qPCR, four miRNAs were differentially expressed in APAs with KCNJ5 mutations compared with those without KCNJ5 mutations. We have recently reported that APAs with KCNJ5 mutations show marked differences in mRNA expression and DNA methylation at the whole genomic level compared to those without KCNJ5 mutations $(8,27)$. In accordance with this finding, Monticone et al. also showed that expression levels of several genes in APAs with KCNJ5 mutations were different from those without KCNJ5 mutations (28). These results strongly support that APAs with KCNJ5 mutations undergo different epigenetic regulation, in mRNA expression, DNA methylation, and miRNA expression, as compared to those without KCNJ5 mutations.

Bioinformatics analysis and qPCR revealed five miRNAs that showed negative correlation between their expression levels and the mRNA levels of their respective target gene: miR34a and CTNND2, ANK2, RIMS3 or MSTN; miR370 and LRRK1 or MAP3K8; miR24 and GRIP1; miR299 and CACNB2; and miR30e and PPP4R4. Although, at present, it is difficult to infer the pathophysiological relevance of CTNND2, ANK2, RIMS3, MSTN, LRRK1, MAP3K8, GRIP1 and PPP4R4 in APA (Table 2), they may be new candidate factors for clarifying the unsolved pathogenic mechanism in PA in the future.

CACNB2 encodes the beta-subunit of the L-type Ca channel $(24,25)$. A previous study showed that CACNB2 upregulation in cardiomyocytes induced activation of Ca signaling, which causes arrhythmia (29). It has been recognized that membrane depolarization and subsequent activation of $\mathrm{Ca}$ signaling play crucial roles in the autonomous aldosterone hypersecretion from APA through increased CYP11B2 expression, a steroidogenic enzyme that is a rate-limiting step in the aldosterone synthesis pathway (14). Somatic mutations of CACNA1D, which encodes the alpha-subunit of L-type Ca channel $(11,12)$, have been shown to be associated 
with APA pathogenesis. Therefore, we further examined the possible relationship between CACNB2 and CYP11B2 using 62 APA tissue specimens. The expression levels of CACNB2 positively correlated to those of CYP11B2 and negatively correlated with those of miR299 in APA tissues. In addition, miR299 levels were negatively correlated to CYP11B2 levels, although bioinformatics analysis did not show any possible interaction between CYP11B2 and miR299. It is, therefore, intriguing to hypothesize that decreased miR299 levels induce CACNB2 expression in APA tissues, which leads to activation of the L-type $\mathrm{Ca}$ channel and subsequent activation of Ca signaling, resulting in increased CYP11B2 expression via the effect of these miRNAs on CACNB2 expression. However, further in vitro studies are required to confirm this hypothesis.

There have been a few studies examining the miRNA expression profiles in APAs $(30,31,32)$. Velázquez-Fernández et al. (30) and He et al. (31) showed that miR-Let7f, 10b, 139-5p, and miR375 were differentially expressed in APAs compared with adjacent adrenal glands or adrenal tissues from idiopathic hyperaldosteronism. Peng et al. reported that miR203 regulated aldosterone production and tumorigenesis in APA, which was determined by comparing APAs with adjacent adrenal gland tissues (32). These discrepancies, including those of the present study, could be explained by the different study design and modality. In particular, our study used ACAs (SHs and NFs) as reference controls, whereas previous studies used adjacent adrenal glands of APAs as reference controls.

In the present study, we set a combination of $\mathrm{SH}$ and $\mathrm{NF}$ as a reference control of APAs. We originally thought that ACAs, except APA and adrenal Cushing syndrome (CS), should be reference controls to APAs. These ACAs include ACAs with weak cortisol secretion, but not showing CS, so-called SH $(33,34)$, and NF. In fact, cases of ACA with $\mathrm{SH}$ have more opportunities to undergo adrenalectomy than those with NFs. Our hierarchical cluster analysis using the whole miRNA sequence data showed that SHs were more closely clustered to NFs than to APAs. In addition, our qPCR analysis revealed that the distribution of miRNAs, which were profiled by Criterion 1 in the whole miRNA sequencing, were comparable between SHs and NFs. We, therefore, considered that it is reasonable to use the combination of $\mathrm{SH}$ and $\mathrm{NF}$ as the reference control to APA.

In the present study, although our whole miRNA sequence did not show statistically significant difference in miR299 levels between APAs and the controls, qPCR analysis showed a significant difference between them. In addition, correlations among miR299, CACNB2, and
CYP11B2 in APAs were also found in entire ACAs. These results are intriguing and suggest that miR299 may be involved in relationship among CACNB2 and CYP11B2, not only in KCNJ5 mutation-positive APAs, but also in the entire APA. However, they were obtained from a sub-analysis based on the negative results of the original screening, and therefore, care should be taken to interpret these data.

There are some limitations in this study. First, our study used correlation analyses of the expression levels of miRNAs and their target genes. Since miRNA can regulate their target gene expression through mRNA degradation and interference with mRNA translation (19), it is possible that our experimental design overlooked target genes for which mRNA translation was inhibited by miRNA. However, the small number of available tumor specimens was not sufficient for additional analysis at the protein level. Secondly, some of our results are based on simple correlation analyses that do not directly prove causality and cannot exclude possible confounding factors. Therefore, additional in vitro studies using cell culture, such as a luciferase assay to confirm CACNB2 as a miR299 target, are required.

In conclusion, we revealed six miRNAs specifically expressed in APAs: differential expression of miR34a and miR370 in APAs compared to control ACAs and differential expression of miR24, 299, 30e, and 93 in APAs with KCNJ5 mutations compared with those without KCNJ5 mutations. We also found a significant correlation in APA tissues among miR299, CACNB2 and CYP11B2 levels, suggesting possible pathophysiological roles of these miRNAs in the dysregulation of $\mathrm{Ca}$ signaling and aldosterone hypersecretion in APAs. The present study postulates a novel concept that miRNA, as an epigenetic factor, plays a key role in the pathogenesis and pathophysiology of APAs. Further studies, including in vitro analyses, are required to clarify the pathophysiological significance of miRNAs in APAs.

\section{Supplementary data}

This is linked to the online version of the paper at https://doi.org/10.1530/ EJE-18-0882.

\section{Declaration of interest}

The authors declare that there is no conflict of interest that could be perceived as prejudicing the impartiality of this study.

\section{Funding}

This work was supported by the Japan Primary Aldosteronism Study (JPAS) supported by a Research Grant from the Japan Agency for Medical Research 
and Development (AMED) (grant number JP17ek0109122, JP18ek0109352) and Grants-in-Aid for Scientific Research from the Ministry of Education, Science, Sports and Culture of Japan (grant number T Y, 16K08962, M M, 16K19392).

\section{Acknowledgements}

The authors thank the staff members of the Molecular Endocrinology and Metabolism Department at Tokyo Medical and Dental University.

\section{References}

1 Funder JW, Carey RM, Mantero F, Murad MH, Reincke M, Shibata H, Stowasser M \& Young WF. The management of primary aldosteronism: case detection, diagnosis, and treatment: an Endocrine Society Clinical Practice guideline. Journal of Clinical Endocrinology and Metabolism 2016101 1889-1916. (https://doi. org/10.1210/jc.2015-4061)

2 Rossi GP, Bernini G, Caliumi C, Desideri G, Fabris B, Ferri C, Ganzaroli C, Giacchetti G, Letizia C, Maccario M et al. A prospective study of the prevalence of primary aldosteronism in 1,125 hypertensive patients. Journal of the American College of Cardiology 200648 2293-2300. (https://doi.org/10.1016/j.jacc.2006.07.059)

3 Milliez P, Girerd X, Plouin PF, Blacher J, Safar ME \& Mourad JJ. Evidence for an increased rate of cardiovascular events in patients with primary aldosteronism. Journal of the American College of Cardiology 200545 1243-1248. (https://doi.org/10.1016/j. jacc.2005.01.015)

4 Rossi GP, Bernini G, Desideri G, Fabris B, Ferri C, Giacchetti G, Letizia C, Maccario M, Mannelli M, Matterello MJ et al. Renal damage in primary aldosteronism: results of the PAPY study. Hypertension 200648 232-238. (https://doi.org/10.1161/01. HYP.0000230444.01215.6a)

5 Williams TA, Lenders JWM, Mulatero P, Burrello J, Rottenkolber M, Adolf C, Satoh F, Amar L, Quinkler M, Deinum J et al. Outcomes after adrenalectomy for unilateral primary aldosteronism: an international consensus on outcome measures and analysis of remission rates in an international cohort. Lancet: Diabetes and Endocrinology 20175 689-699. (https://doi.org/10.1016/S2213-8587(17)30135-3)

6 Lenzini L, Caroccia B, Campos AG, Fassina A, Belloni AS, Seccia TM, Kuppusamy M, Ferraro S, Skander G, Bader M et al. Lower expression of the TWIK-related acid-sensitive K+channel 2 (TASK-2) gene is a hallmark of aldosterone-producing adenoma causing human primary aldosteronism. Journal of Clinical Endocrinology and Metabolism 2014 99 E674-E682. (https://doi.org/10.1210/jc.2013-2900)

7 Williams TA, Monticone S, Crudo V, Warth R, Veglio F \& Mulatero P. Visinin-like 1 is upregulated in aldosterone-producing adenomas with KCNJ5 mutations and protects from calcium-induced apoptosis. Hypertension 201259 833-839. (https://doi.org/10.1161/ HYPERTENSIONAHA.111.188532)

8 Murakami M, Yoshimoto T, Nakabayashi K, Nakano Y, Fukaishi T, Tsuchiya K, Minami I, Bouchi R, Okamura K, Fujii Y et al. Molecular characteristics of the KCNJ5 mutated aldosterone-producing adenomas. Endocrine-Related Cancer 201724 531-541. (https://doi. org/10.1530/ERC-17-0117)

9 Choi M, Scholl UI, Yue P, Björklund P, Zhao B, Nelson-Williams C, Ji W, Cho Y, Patel A, Men CJ et al. K+ channel mutations in adrenal aldosterone-producing adenomas and hereditary hypertension. Science 2011331 768-772. (https://doi.org/10.1126/science.1198785)

10 Beuschlein F, Boulkroun S, Osswald A, Wieland T, Nielsen HN, Lichtenauer UD, Penton D, Schack VR, Amar L, Fischer E et al. Somatic mutations in ATP1A1 and ATP2B3 lead to aldosteroneproducing adenomas and secondary hypertension. Nature Genetics 201345 440.e1-444.e1. (https://doi.org/10.1038/ng.2550)
11 Azizan EAB, Poulsen H, Tuluc P, Zhou J, Clausen MV, Lieb A, Maniero C, Garg S, Bochukova EG, Zhao W et al. Somatic mutations in ATP1A1 and CACNA1D underlie a common subtype of adrenal hypertension. Nature Genetics 201345 1055-1060. (https://doi. org/10.1038/ng.2716)

12 Scholl UI, Goh G, Stölting G, De Oliveira RC, Choi M, Overton JD, Fonseca AL, Korah R, Starker LF, Kunstman JW et al. Somatic and germline CACNA1D calcium channel mutations in aldosteroneproducing adenomas and primary aldosteronism. Nature Genetics 201345 1050-1054. (https://doi.org/10.1038/ng.2695)

13 Murakami M, Yoshimoto T, Minami I, Bouchi R, Tsuchiya K, Hashimoto K, Izumiyama H, Fujii Y, Endo T, Akashi T et al. A novel somatic deletion mutation of ATP2B3 in aldosterone-producing adenoma. Endocrine Pathology 201526 328-333. (https://doi. org/10.1007/s12022-015-9400-9)

14 Zennaro MC, Jeunemaitre X \& Boulkroun S. Integrating genetics and genomics in primary aldosteronism. Hypertension $201260580-588$. (https://doi.org/10.1161/HYPERTENSIONAHA.111.188250)

15 Howard B, Wang Y, Xekouki P, Faucz FR, Jain M, Zhang L, Meltzer PG, Stratakis CA \& Kebebew E. Integrated analysis of genome-wide methylation and gene expression shows epigenetic regulation of CYP11B2 in aldosteronomas. Journal of Clinical Endocrinology and Metabolism 201499 E536-E543. (https://doi. org/10.1210/jc.2013-3495)

16 Yoshii Y, Oki K, Gomez-Sanchez CE, Ohno H, Itcho K, Kobuke K \& Yoneda M. Hypomethylation of CYP11B2 in aldosterone-producing adenoma. Hypertension 201668 1432-1437. (https://doi.org/10.1161/ HYPERTENSIONAHA.116.08313)

17 Bartel DP. MicroRNAs: genomics, biogenesis, mechanism, and function. Cell 2004116 281-297. (https://doi.org/10.1016/S00928674(04)00045-5)

18 Bartel DP. MicroRNAs: target recognition and regulatory functions. Cell 2009136 215-233. (https://doi.org/10.1016/j.cell.2009.01.002)

19 Morozova N, Zinovyev A, Nonne N, Pritchard LL, Gorban AN \& Harel-Bellan A. Kinetic signatures of microRNA modes of action. RNA 201218 1635-1655. (https://doi.org/10.1261/rna.032284.112)

20 Croce CM. Causes and consequences of microRNA dysregulation in cancer. Nature Reviews: Genetics 200910 704-714. (https://doi. org/10.1038/nrg2634)

21 Callegari E, Gramantieri L, Domenicali M, D'Abundo L, Sabbioni S \& Negrini M. MicroRNAs in liver cancer: a model for investigating pathogenesis and novel therapeutic approaches. Cell Death and Differentiation 201522 46-57. (https://doi.org/10.1038/ cdd.2014.136)

22 Murakami M, Yoshimoto T, Nakabayashi K, Tsuchiya K, Minami I, Bouchi R, Izumiyama H, Fujii Y, Abe K, Tayama C et al. Integration of transcriptome and methylome analysis of aldosterone-producing adenomas. European Journal of Endocrinology 2015173 185-195. (https://doi.org/10.1530/EJE-15-0148)

23 Robinson MD, McCarthy DJ \& Smyth GK. edgeR: a bioconductor package for differential expression analysis of digital gene expression data. Bioinformatics 201026 139-140. (https://doi.org/10.1093/ bioinformatics/btp616)

24 Hong GL, Chen XZ, Liu Y, Liu YH, Fu X, Lin SB \& Zhu Q. Genetic variations in MOV10 and CACNB2 are associated with hypertension in a Chinese Han population. Genetics and Molecular Research 201312 6220-6227. (https://doi.org/10.4238/2013.December.4.9)

25 Qi ZL, Kobrinsky E, Harry JB, Ravindran A \& Soldatov NM. New determinant for the Cav $\beta 2$ subunit modulation of the Cav1.2 calcium channel. Journal of Biological Chemistry 2008283 15577-15588. (https://doi.org/10.1074/jbc.M802035200)

26 Tiedt S, Prestel M, Malik R, Schieferdecker N, Duering M, Kautzky V, Stoycheva I, Böck J, Northoff BH, Klein M et al. RNA-seq identifies circulating MIR-125a-5p, MIR-125b-5p, and MIR-143-3p as potential biomarkers for acute ischemic stroke. Circulation Research 2017121 970-980. (https://doi.org/10.1161/CIRCRESAHA.117.311572) 
27 Murakami M, Yoshimoto T, Nakano Y, Tsuchiya K, Minami I, Bouchi R, Fujii Y, Nakabayashi K, Hashimoto K, Hata KI et al. Expression of inflammation-related genes in aldosterone-producing adenomas with KCNJ5 mutation. Biochemical and Biophysical Research Communications 2016476 614-619. (https://doi.org/10.1016/j. bbrc.2016.06.007)

28 Monticone S, Hattangady NG, Nishimoto K, Mantero F, Rubin B, Cicala MV, Pezzani R, Auchus RJ, Ghayee HK, Shibata H et al. Effect of KCNJ5 mutations on gene expression in aldosterone-producing adenomas and adrenocortical cells. Journal of Clinical Endocrinology and Metabolism 201297 E1567-E1572. (https://doi.org/10.1210/ jc.2011-3132)

29 Zhang Q, Chen J, Qin Y, Wang J \& Zhou L. Mutations in voltagegated L-type calcium channel: implications in cardiac arrhythmia. Channels 201812 201-218. (https://doi.org/10.1080/19336950.2018. 1499368)

30 Velázquez-Fernández D, Caramuta S, Özata DM, Lu M, Höög A, Bäckdahl M, Larsson C, Lui WO \& Zedenius J. MicroRNA expression patterns associated with hyperfunctioning and nonhyperfunctioning phenotypes in adrenocortical adenomas. European Journal of Endocrinology 2014170 583-591. (https://doi.org/10.1530/ EJE-13-0817)

31 He J, Cao Y, Su T, Jiang Y, Jiang L, Zhou W, Zhang C, Wang W \& Ning G. Downregulation of miR-375 in aldosterone-producing adenomas promotes tumour cell growth via MTDH. Clinical Endocrinology 201583 581-589. (https://doi.org/10.1111/cen.12814)

32 Peng KY, Chang HM, Lin YF, Chan CK, Chang CH, Chueh S-CJ, Yang SY, Huang KH, Lin YH, Wu VC et al. MicroRNA-203 modulates aldosterone levels and cell proliferation by targeting Wnt5a in aldosteroneproducing adenomas. Journal of Clinical Endocrinology and Metabolism 2018103 3737-3747. (https://doi.org/10.1210/jc.2018-00746)

33 Di Dalmazi G, Pasquali R, Beuschlein F \& Reincke M. Subclinical hypercortisolism: a state, a syndrome, or a disease? European Journal of Endocrinology 2015173 M61-M71. (https://doi.org/10.1530/EJE-15-0272)

34 Chiodini I. Clinical review: diagnosis and treatment of subclinical hypercortisolism. Journal of Clinical Endocrinology and Metabolism 201196 1223-1236. (https://doi.org/10.1210/jc.2010-2722)

$35 \mathrm{Lu}$ Q, Aguilar BJ, Li M, Jiang Y \& Hua YH. Genetic alterations of $\delta$-catenin/NPRAP/Neurojungin (CTNND2): functional implications in complex human diseases. Human Genetics 2016135 1107-1116. (https://doi.org/10.1007/s00439-016-1705-3)

36 Lu Q, Paredes M, Medina M, Zhou J, Cavallo R, Peifer M, Orecchio L $\&$ Kosik KS. $\delta$-Catenin, an adhesive junction-associated protein which promotes cell scattering. Journal of Cell Biology 1999144 519-532. (https://doi.org/10.1083/jcb.144.3.519)

37 Mohler PJ, Anthony O \& Bennett V. Ankyrins. Journal of Cell Science 2002115 1565-1566.

38 Wang Y, Sugita S \& Su TC. The RIM/NIM family of neuronal C 2 domain proteins. Journal of Biological Chemistry 2000275 20033-20044. (https://doi.org/10.1074/jbc.M909008199)

39 Mcpherron AC, Lawlert AM \& Lee SJ. Regulation of skeletal muscle mass in mice by a new TGF-fl superfamily member. Nature 1997387 83-90. (https://doi.org/10.1038/387083a0)

40 Hanafusa H, Ishikawa K, Kedashiro S, Saigo T, Iemura S, Natsume T, Komada M, Shibuya H, Nara A \& Matsumoto K. Leucine-rich repeat kinase LRRK1 regulates endosomal trafficking of the EGF receptor. Nature Communications 20112 158. (https://doi.org/10.1038/ ncomms1161)

41 Gantke T, Sriskantharajah S \& Ley SC. Regulation and function of TPL-2, an IB kinase-regulated MAP kinase kinase. Cell Research 2011 21 131-145. (https://doi.org/10.1038/cr.2010.173)

42 Van Acker GJD, Perides G, Weiss ER, Das S, Tsichlis PN \& Steer ML. Tumor progression locus- 2 is a critical regulator of pancreatic and lung inflammation during acute pancreatitis. Journal of Biological Chemistry 2007282 22140-22149. (https://doi.org/10.1074/jbc. M702225200)

43 Mao L, Takamiya K, Thomas G, Lin DT \& Huganir RL. GRIP1 and 2 regulate activity-dependent AMPA receptor recycling via exocyst complex interactions. PNAS 2010107 19038-19043. (https://doi. org/10.1073/pnas.1013494107)

44 Tan HL, Queenan BN \& Huganir RL. GRIP1 is required for homeostatic regulation of AMPAR trafficking. PNAS 2015112 10026-10031. (https://doi.org/10.1073/pnas.1512786112)

45 Chen GI, Tisayakorn S, Jorgensen C, Ambrosio LMD, Goudreault M \& Gingras AC. PP4R4/KIAA1622 forms a novel stable cytosolic complex with phosphoprotein phosphatase 4. Journal of Biological Chemistry 2008283 29273-29284. (https://doi.org/10.1074/jbc. M803443200)

Received 1 November 2018

Revised version received 3 May 2019

Accepted 14 May 2019 